\title{
Microbial and Immune Biomarkers in Infants at Risk for Allergies Fed an Intact Cow's Milk Formula Containing Specific Non-Digestible Carbohydrates
}

\author{
Karen Knipping ${ }^{1,2 *}$, Anna Orsi ${ }^{3}$, Günther Boehm ${ }^{1,4}$, Francesca Castoldi ${ }^{5}$, Johan Garssen ${ }^{1,2}$, Maria Giannì ${ }^{3}$, Tom Groot Kormelink ${ }^{2}$, Gianluca \\ Lista $^{5}$, Paola Marangione ${ }^{5}$, Frank Redegeld ${ }^{2}$, Paola Roggero ${ }^{3}$ and Fabio Mosca ${ }^{3}$ \\ ${ }^{1}$ Danone Research-Centre for Specialised Nutrition, Wageningen, The Netherlands \\ ${ }^{2}$ Utrecht Institute for Pharmaceutical Sciences, Utrecht University, Utrecht, The Netherlands \\ ${ }^{3}$ Fondazione IRCCS Cà Granda Ospedale Maggiore Policlinico, Department of Maternal and Pediatric Sciences, University of Milan, Italy \\ ${ }^{4}$ Sophia Children's Hospital, Erasmus University, Rotterdam, The Netherlands \\ ${ }^{5} \mathrm{NICU}$, "V.Buzzi" Children's Hospital, ICP, Milan, Italy
}

\section{Abstract}

Background: Oligosaccharides may support postnatal immune development by influencing the constitution of gastrointestinal microbiota. This prospective, double-blind, randomised, placebo controlled trial investigated the effect of a specific prebiotic mixture of short chain galactooligosaccharides (scGOS) and long chain fructooligosaccharides (ICFOS) on microbiota and immune biomarkers during the first six months of life in high risk infants for allergies fed a formula based on intact cow's milk protein.

Methods: If formula feeding was started, the infant was randomly assigned to one of two cow's milk formula groups $(0.8 \mathrm{~g} / 100 \mathrm{ml} \mathrm{scGOS/lcFOS}$ or maltodextrine as control). The faecal microbiota of the scGOS/lcFOS and control groups was analysed. In a subgroup blood was collected at the age of six months for serum biomarkers. A reference group consisted of 90 exclusively breast fed infants up to six months of age.

Results: In both the prebiotic group and control group a total of 51 infants completed the study. The scGOS/lcFOS supplementation was associated with a significantly higher number of faecal bifidobacteria and lactobacilli counts compared to controls, accompanied by significantly lower $\mathrm{pH}$ values in the faeces. In the serum, the scGOS/lcFOS group showed a trend towards a decrease in total IgE levels as well as a trend towards a decrease in the percentage of children with elevated (>15 kU/I) IgE. There were no differences found in kappa Ig-fLC and lambda Ig-fLC between the two groups.

Conclusions: scGOS/IcFOS administration significantly influences the composition of bifidobacteria and lactobacilli. There were some observations with respect to the immune parameters which need further investigation.

Keywords: Oligosaccharides; Bifidobacteria; Lactobacilli; Ig-fLC; IgE

Abbreviations: lcFOS: Long Chain Fructooligosaccharides; scGOS: Short Chain Galactooligosaccharides; Ig-fLC: Immunoglobulin Free Light Chain

\section{Background}

In Italy, as in many European countries, the feeding of an infant formula based on hydrolysed cow's milk protein is recommended for infants with a family history of atopy or allergies [1]. However, studies analysing the preventive effect of hydrolysed formula in comparison to formula based on intact cow's milk provide only limited evidence that feeding with hydrolysed formula reduces allergies in infants and children [2]. Consequently, other strategies than the avoidance of nutritive antigens are under discussion [3]. Such new strategies may include the modification of intestinal microbiota as an important factor for early modulation of the immune system leading to a lower susceptibility for allergy development early and later in life.

Recently, Moro et al. reported a significant reduction in the cumulative incidence of atopic dermatitis at 6 months [4] and 2 years of age [5] in high risk infants for allergies fed an extensively hydrolysed formula supplemented with prebiotics (scGOS/lcFOS in a ratio 9:1 at a concentration of $0.8 \mathrm{~g} / 100 \mathrm{ml}$ ) in comparison to infants fed the same formula but supplemented with a placebo. This was accompanied by a plasma antibody profile that was suggestive for a lower susceptibility to allergy development [6]. This was in line with experimental data obtained in mice with this scGOS/lcFOS mixture, indicating its immune modulatory capacity in both respiratory as well as food and skin allergic reactions $[7,8]$. In addition to the traditional biomarkers for allergy, such as IgE which predicts and is associated with atopic dermatitis in at least $60 \%$ of the atopic dermatitis cases, Ig-fLC was analysed as well. Recent data indicated the high relevance of both IgE as well as Ig-fLC in both atopic dermatitis and allergy to cow's milk proteins [9].

In this first explorative study it was investigated whether the immune modulatory capacity of a specific scGOS/lcFOS mixture can influence the occurrence and severity of atopic dermatitis, microbiota composition, serum antibody and Ig-fLC profiles in high risk infants for allergies fed a formula based on intact cow's milk protein, in contrast to the current recommendation to feed these infants infant formulas based on hydrolysed cow's milk protein.

*Corresponding author: Karen Knipping, Danone Research Centre fo Specialised Nutrition, P.O. Box 7005, 6700 CA Wageningen, The Netherlands, E-mail: karen.knipping@danone.com

Received July 18, 2012; Accepted August 23, 2012; Published August 25, 2012

Citation: Knipping K, Orsi A, Boehm G, Castoldi F, Garssen J, et al. (2012 Microbial and Immune Biomarkers in Infants at Risk for Allergies Fed an Intact Cow's Milk Formula Containing Specific Non-Digestible Carbohydrates. J Nutr Disorders Ther 2:119. doi:10.4172/2161-0509.1000119

Copyright: (c) 2012 Knipping K, et al. This is an open-access article distributed under the terms of the Creative Commons Attribution License, which permits unrestricted use, distribution, and reproduction in any medium, provided the original author and source are credited. 


\section{Subjects and Methods}

\section{Study population}

The study has been performed in the Clinica Mangiagalli, University Medical School of Milan and in the "V. Buzzi" Children's HospitalICP of Milan from March 2005 until March 2008. The study protocol was approved by the Ethic Committees of both participating hospitals and written informed consent was obtained from each family before enrolment in the study. According to the policy of the two hospitals breast feeding was strongly recommended to each mother. The parents were informed about the study at discharge from the maternity unit and were asked to contact the hospital if they started formula feeding. In the formula groups, mixed feeding (breast feeding and formula feeding) was accepted until the sixth week of life provided that the start of formula feeding was before the end of the second week of life. In cases where the mother continued partial breast feeding for more than six weeks the infant was excluded from the study.

Inclusion criteria were: healthy, term infants (gestational age 37-42 weeks), with a normal birth weight ( $>10$ percentile for gestational age according to the WHO growth charts), infants whose mother had to start formula feeding due to lack of sufficient amounts who were not older than two weeks of age when entering the study, at high risk to develop an atopic disease (both parents or one parent and one sibling with manifest atopic symptoms of hay fever, asthma or atopic dermatitis). Exclusion criteria were: infants with known congenital diseases or chromosomal abnormalities, cardiac, respiratory, gastrointestinal or other systemic diseases, severe brain disease and study pre-feedings of the infants which could interfere with the study (e.g. non-cow's milk based formulas, hypo-allergenic formulas, probiotic formulas), infants with actual or previous illnesses which could interfere with the study.

\section{Trial design}

The study was a double-blind, placebo-controlled, randomized prospective study with parallel group design. Enrolment was started at the infants' age of two weeks. On enrolment infants were randomly allocated to receive scGOS/lcFOS or control formula for six months. The composition of both formulas was similar and based on intact cow's milk protein with a whey/casein ratio of 60:40 (Table 1). The test formulas were supplemented either with a mixture of scGOS/lcFOS (ratio 9:1; concentration $0.8 \mathrm{~g} / 100 \mathrm{ml}$ or with the same quantity of maltodextrine as control). Randomization of the formula groups was by means of a random numbers table and the blinding was maintained by coding the two trial formulas with the suffix " $\mathrm{N}$ " or "O" to the product name. The allocation of the two blinded formulas to the individual infants was performed by persons not involved in the study (hospital pharmacy). Both the investigators and the infants' parents were blinded to the formulas. The infants received the starting formula until the age of six months. No solid foods were allowed before the fifth month of infants' age. The total treatment period started on day of enrolment and lasted up to the completed six months of infants' age. Both formula and breast fed infants were fed ad libitum. The infants underwent follow up visits at one, three and six months. During the visits the parents were interviewed for nutrition history and the appearance of skin symptoms.

\section{Growth parameters}

For all infants, growth parameters were measured at birth and at each study visit. Body weight was measured by a scale with an accuracy of $\pm 5 \mathrm{~g}$. Recumbent length was measured on a Harpenden stadiometer to the nearest $1 \mathrm{~mm}$. The head circumference was measured using a non-stretch measuring tape to the nearest $1 \mathrm{~mm}$.

\section{Atopic dermatitis examination}

During the visits at one, three and six months of age the infants' skin was examined and screened for symptoms of atopic dermatitis. According to the recommendation by Harrigan and Rabinowitz [10] and Muraro et al. [11] the infants' skin was examined in the face, skull facial and/or extensor part of the extremities. Additionally, the appearance of pruritus and duration of the symptoms of at least four weeks has been used as basis for the diagnosis of atopic dermatitis. The intensity of the alteration of the skin was scored by the SCORAD index as recommended by the European Task Force on AD [12,13].

\section{Fecal sample collection and microbial analyses}

During the visits at one, three and six months of age a fresh sample of stool was obtained in the scGOS/lcFOS and control groups for $\mathrm{pH}$ measurement and microbiological analysis. By request of the Ethic Committees no faecal samples have been obtained from the breast fed reference group. The samples were homogenised in a cryoprotective transport medium as previously described [14]. For identification of bifidobacteria and lactobacilli commercially available selective media were used as described previously [14]. The numbers are presented as colony forming units (CFU)/g stool. The $\mathrm{pH}$ was measured using a $\mathrm{pH}$ meter equipped with a glass capillary.

\begin{tabular}{|c|c|c|c|}
\hline Formula: & & scGOS/lcFOS & Control \\
\hline Fat & (g) & 3.6 & 3.6 \\
\hline Carbohydrates & (g) & 7.2 & 8.2 \\
\hline - Lactose & (g) & 7.2 & 7.2 \\
\hline - scGOS/IcFOS 9:1 & (g) & 0.8 & 0 \\
\hline - Maltodextrine & (g) & 0 & 0.8 \\
\hline Protein & (g) & 1,5 & 1,5 \\
\hline whey/casein ratio & & $60 / 40$ & $60 / 40$ \\
\hline Minerals & (g) & 0.4 & 0.4 \\
\hline$-\mathrm{Na}$ & (g) & 0.02 & 0.02 \\
\hline$-K$ & (mg) & 82 & 82 \\
\hline - Ca & $(\mathrm{mg})$ & 66 & 66 \\
\hline$-M g$ & (mg) & 5.2 & 5.2 \\
\hline$-P$ & (mg) & 42 & 42 \\
\hline$-\mathrm{Cl}$ & (mg) & 53 & 53 \\
\hline$-J$ & $(\mu g)$ & 10 & 10 \\
\hline - Cu & (mg) & 0.04 & 0.04 \\
\hline - Zn & $(\mathrm{mg})$ & 0.5 & 0.5 \\
\hline$-M n$ & $(\mathrm{mg})$ & 0.01 & 0.01 \\
\hline \multicolumn{4}{|l|}{ Vitamins } \\
\hline - Vit. A & $(\mu \mathrm{g})$ & 60 & 60 \\
\hline - Vit. B1 & $(\mathrm{mg})$ & 0.04 & 0.04 \\
\hline - Vit. B2 & (mg) & 0.12 & 0.12 \\
\hline - Vit. B6 & (mg) & 0.04 & 0.04 \\
\hline - Niacine amid & $(\mathrm{mg})$ & 0.7 & 0.7 \\
\hline - Vit. C & $(\mathrm{mg})$ & 8 & 8 \\
\hline - Vit. D3 & $(\mu \mathrm{g})$ & 1 & 1 \\
\hline - Vit. E & (mg) & 0.6 & 0.6 \\
\hline - Biotin & (mg) & 0.001 & 0.001 \\
\hline - Ca-D-pantothenate & (mg) & 0.4 & 0.4 \\
\hline - Vit. K1 & $(\mu \mathrm{g})$ & 0.003 & 0.003 \\
\hline - Folic acid & $(\mu \mathrm{g})$ & 10 & 10 \\
\hline - Vit. B12 & $(\mu \mathrm{g})$ & 0.2 & 0.2 \\
\hline Osmolarity & $(\mathrm{mOsmol} / \mathrm{l})$ & $280-290$ & $280-290$ \\
\hline Energy content & (kcal) & 67 & 71 \\
\hline Standard solution & $(\%)$ & 13 & 13 \\
\hline
\end{tabular}

Table 1: Composition of the study formulas. 


\section{Serum sample collection}

In a subgroup of 63 infants from the scGOS/lcFOS and control groups, parents allowed obtaining $0.5 \mathrm{ml}$ blood at the age of six months. By request of the Ethic Committees no serum samples have been obtained from the breast fed reference group. The blood was collected in a test tube and centrifuged (1300 RCF for 10 minutes). The separated serum was immediately frozen and stored at $-80^{\circ} \mathrm{C}$ until analysis.

\section{Serum total and $\beta$-lactoglobulin-specific IgE and IgG4}

For measurements of total IgE and IgG4 as well as ß-lactoglobulinspecific IgE and IgG4 in serum the ImmunoCAP $100^{\circ}$ system, an automated allergy-testing system from Phadia AB (Uppsala, Sweden), was used [15]. For total and B-lactoglobulin-specific IgG4, the serum samples were pre-diluted a 100 times in specific IgA/IgG sample diluent. The tests were performed according to the manufacturer's instructions, total and specific IgE results are expressed in $\mathrm{kU} / \mathrm{L}$, total and specific IgG4 in mgA/L. Total and specific IgE antibody values $\geq$ $0.35 \mathrm{kU} / \mathrm{L}$ and total and specific IgG4 antibody values $\geq 0.07 \mathrm{mgA} / \mathrm{L}$ were considered positive.

\section{Degranulation of RBL cells with human receptor (RBL-Heia- 2B12 Cells)}

The a-chain of human Fce receptor type 1 (hFceR1) complex was transfected into the rat basophilic leukaemia (RBL) cell line RBL$2 \mathrm{H} 3$ to establish the stable transfected cell-line RBL-hEIa-2B12, a kind gift of Dr. Teshima, Japan [16]. The functional degranulation capacity of RBL-hEIa-2B12 cells in response to stimulation with cow's milk allergens was tested as well as a functional readout for IgE as described previously [17]. In short, RBL-hEIa-2B12 cells $\left(1 \times 10^{5} /\right.$ well $)$ were sensitized with $5 \mu \mathrm{g} / \mathrm{ml}$ human purified $\mathrm{IgE}$ or with serum of the individual participants (1:50). Cells sensitized with human purified IgE were stimulated with anti-human $\operatorname{IgE}$ antibody $(5 \mu \mathrm{g} / \mathrm{mL})$ for 1 hour and served as maximum amount of release. Cells sensitized with serum were stimulated with milk powder $(1 \mu \mathrm{g} / \mathrm{mL})$ for 1 hour. The $ß$-hexosaminidase activity released into the medium was determined by a fluorescence assay using 4-methylumbelliferyl-N-acetyl- $\alpha-\mathrm{D}$ glucosamine as a substrate. The $ß$-hexosaminidase activity released into the medium is expressed as a percentage of maximum amounts of release.

\section{Serum immunoglobulin free light chain}

Total kappa and lambda immunoglobulin-free light chain (Ig-fLC) serum concentrations were determined using an ELISA adapted from Abe et al. [18]. In short, plates were coated o/n with goat-anti mouse $\operatorname{IgG}(4 \mu \mathrm{g} / \mathrm{mL})$, blocked for 1 hour (RT) and subsequently incubated with mouse-anti human kappa or lambda Ig-fLC $(1 \mu \mathrm{g} / \mathrm{mL}$; Fk-C8 or F入-G9) for 1 hour. After incubation with different dilutions of serum samples and standards for 1 hour, plates were incubated with HRPlabelled goat $\left.\mathrm{F}(\mathrm{ab})_{2}\right)_{2}$-anti human kappa or lambda Ig-fLC (1:20.000) for 1 hour. Finally, TMB substrate was added and the enzymatic reaction was terminated by adding $0.9 \mathrm{M} \mathrm{H}_{2} \mathrm{SO}_{4}$. Between incubation steps, wells were washed three times with PBS-T ( $0.1 \%$ tween-20). Per sample, at least three data points were used to calculate the concentration.

\section{Statistical Analysis}

All analyses were performed using the statistical software package SPSS 15.0. Normal distribution was tested using the Shapiro-Wilk test and variances using the Levene test. When the data were normally distributed and the variances were equal, a two-sample t-test was used to compare groups. When variances were not equal, the Welch test was used to compare groups. Comparison of percentages was done by using a two-sample Pearson Chi-Square test. Values of P less than 0.05 were considered significant.

\section{Results}

\section{Study population}

Out of 535 children who were screened, 380 met the entry criteria and were enrolled in the study. 180 infants were enrolled in the breast fed reference group and 200 infants were randomized to treatment, 100 in the scGOS/lcFOS group and 100 in the control group. In cases where the mother continued partial breast feeding for more than 6 weeks the infant was excluded from the study ( $n=30$ infants in the scGOS/ lcFOS group, $n=35$ in the control group). Due to 19 drop-outs in the scGOS/lcFOS group and 14 drop-outs in the control group, 51 infants completed the study in both the scGOS/lcFOS and control groups. In the reference group, 90 exclusively breast fed infants up to six months of age completed the study (Figure 1).

\section{Growth parameters}

There were no differences found in weight, length and head circumference between the formulas and breast fed infants (data not shown).

\section{Atopic dermatitis and SCORAD}

There were no significant differences observed in cumulative incidences of AD or SCORAD scores between the scGOS/lcFOS (1 month $4.2 \%$; 3 months $20.8 \%$; 6 months $29.1 \%$ ), control (1 month $15.7 \%$; 3 months $25.5 \%$; 6 months $29.4 \%$ ) and the breast fed reference group (1 month 0\%; 3 months 22.2\%; 6 months 30.0\%) (Figure 2).

\section{Fecal counts of Bifidobacteria and Lactobacilli and $\mathrm{pH}$ levels}

In the scGOS/lcFOS group, a significantly higher number of bifidobacteria and lactobacilli were found at all time points when compared to the control group. This was accompanied by significantly lower $\mathrm{pH}$ values in the faeces of the infants fed the prebiotic formula (Figure 3).

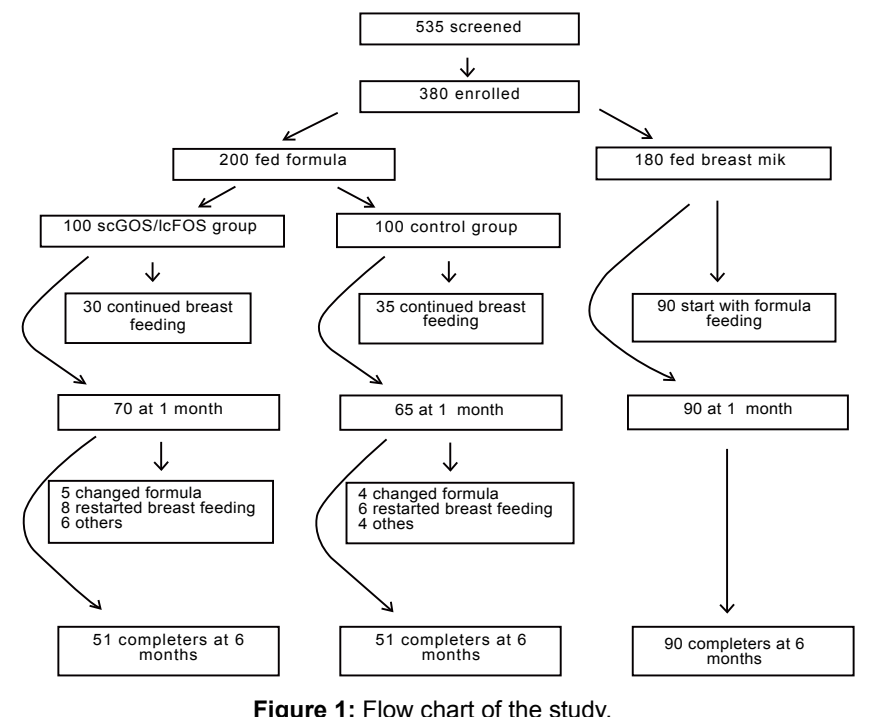

Figure 1: Flow chart of the study. 


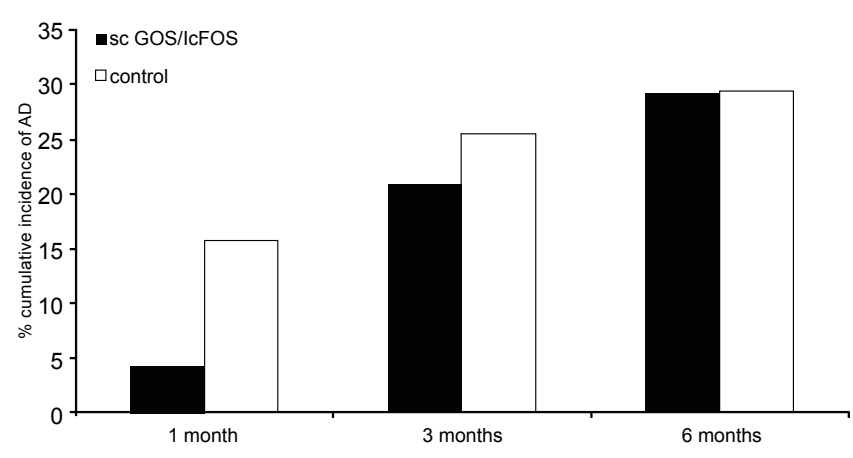

Figure 2: Cumulative incidence of atopic dermatitis at 1,3 and 6 months of age in percentages in the group fed a formula supplemented with scGOS/ IcFOS or maltodextrine as control.

(A) Bifidobacteria

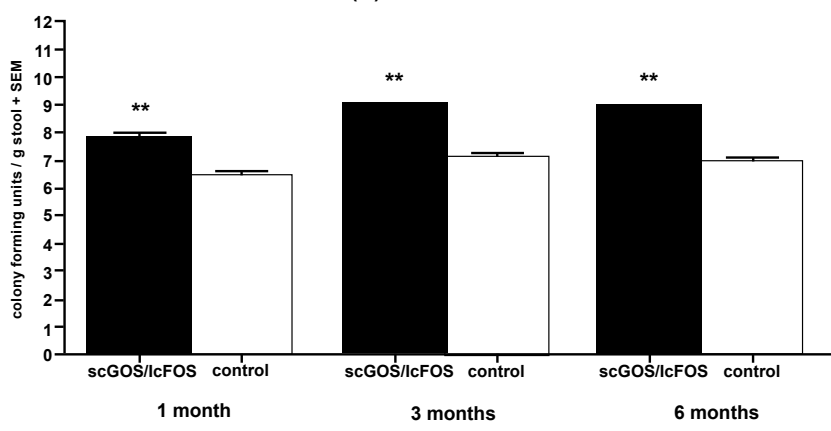

(B) Lactobacilli

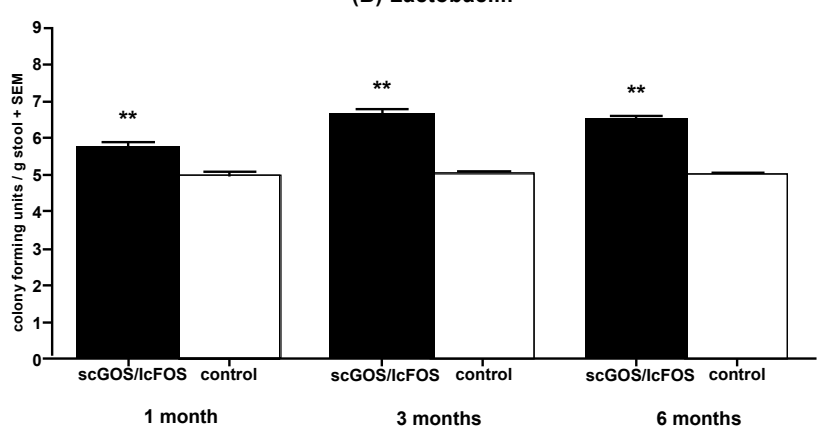

(C) $\mathrm{pH}$

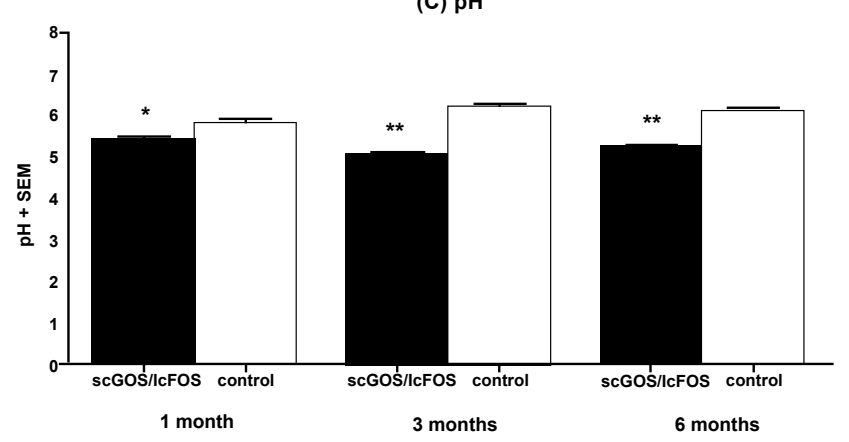

Figure 3: Faecal counts in colony forming units/g stool of bifidobacteria (A) and lactobacilli $(B)$ as well as $\mathrm{pH}$ levels $(\mathrm{C})$ at 1,3 and 6 months of age. Data is represented as mean $+\mathrm{SD}$. * $\mathrm{p}<0.05 ;{ }^{* *} \mathrm{p}<0.01$
Serum total and $\beta$-Lactoglobulin-specific Ige/Igg4, functional degranulation capacity of RBL-Heia-2B12 cells and immunoglobulin free light chain

In serum, the scGOS/lcFOS group showed a trend towards a decrease in total IgE levels (scGOS/lcFOS $8.4 \mathrm{kU} / \mathrm{L}$; control 17.2 $\mathrm{kU} / \mathrm{L} ; \mathrm{p}=0.06$ ) as well as a trend towards a decrease in the percentage of children with elevated (>15 kU/l) IgE levels (scGOS/lcFOS $13.6 \%$; control 28.6\%; $p=0.1$ ) (Figure 4). The concentration of B-lactoglobulinspecific IgE was low $(<0.35 \mathrm{kU} / \mathrm{L})$ in both groups and showed no differences (data not shown). When RBL-hEIa-2B12 cells were sensitized with the serum of the individual participants, none of the sera was able to induce degranulation of the RBL cells after incubation with milk powder, indicating an absence of functional cow's milkspecific IgE in the sera (data not shown).

No significant differences were found in total IgG4 (scGOS/lcFOS $55.7 \mathrm{mgA} / \mathrm{L}$; control $59.3 \mathrm{mgA} / \mathrm{L}$ ) and B-lactoglobulin-specific IgG4 (scGOS/lcFOS $10.9 \mathrm{mgA} / \mathrm{L}$; control $16.9 \mathrm{mgA} / \mathrm{L}$ ) or in the ratio IgG4/ $\operatorname{IgE}$ between the two groups (data not shown).

No significant differences were found in the amount of kappa IgfLC (scGOS/lcFOS $8.93 \mu \mathrm{g} / \mathrm{mL}$; control $9.95 \mu \mathrm{g} / \mathrm{mL}$ ) and lambda IgfLC (scGOS/lcFOS $14.02 \mu \mathrm{g} / \mathrm{mL}$; control $15.57 \mu \mathrm{g} / \mathrm{mL}$ ), or in the ratio lambda Ig-fLC/ kappa Ig-fLC, between the two groups (Figure 5).

\section{Discussion}

In the present exploratory study, the effect of dietary modulation of the intestinal microbiota and immune biomarkers related to atopic dermatitis was investigated in children at risk for allergies since they all
(A) total IgE

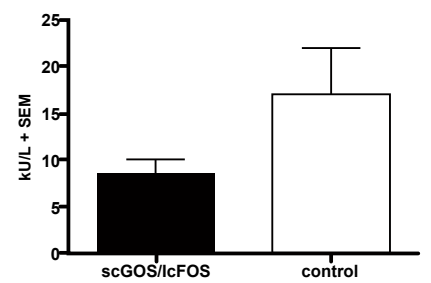

(B) elevated IgE

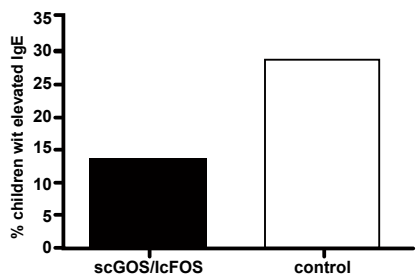

Figure 4: Serum total $\lg \mathrm{E}$ concentration in $\mathrm{kU} / \mathrm{L}(\mathrm{A})$ and elevated total $\lg \mathrm{E}$ in percentages (B), collected at the age of 6 months. Data is represented as mean $+S D$.

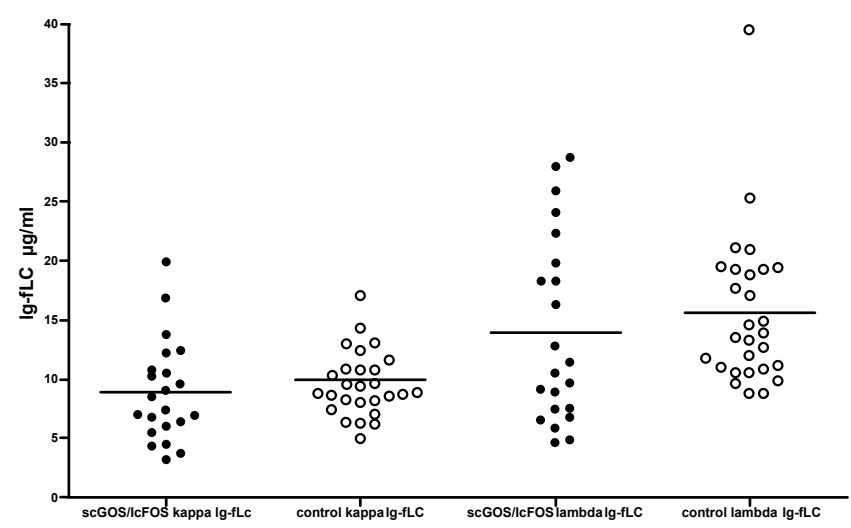

Figure 5: Serum kappa-fLC and lambda-fLC in $\mu \mathrm{g} / \mathrm{ml}$, collected at the age of 6 months. Data is represented as individual data points (dots) and mean (line) 
have a family history of atopy. The infants were fed an infant formula based on intact cow's milk protein. Although it was realized that the study was underpowered regarding atopic dermatitis comparisons, the study was aimed at exploratory comparisons of biomarkers and did indicate some significant effects as well as some trends. In the study described by Moro et al. [4], high risk infants for allergies were fed an extensively hydrolysed formula supplemented with the same prebiotic mixture as used in the current study. Here, in infants at six months of age the atopic dermatitis incidence was significantly lower in the scGOS/lcFOS group compared to the control. In contrast, in this current study there is only a trend to fewer children with atopic dermatitis-like symptoms at one month of age. This trend however disappeared over time at the age of six months. This might be due to the significantly higher antigenic load in total cow's milk protein formula. If the total protein level is higher and thus the antigenic load is higher it might be more difficult to affect the response by prebiotic administration. In the case of extensive hydrolysates the antigenic load is much lower leading to less strong adverse immune reactions and, as a consequence, a higher sensitivity for prebiotic induced immune modulation. In future studies with intact cow's milk proteins in children at risk for allergies it might be beneficial to add another immunomodulatory component to the scGOS/lcFOS mixture. Addition of pectin-derived acidic oligosaccharides (pAOS) to the scGOS/lcFOS mixture in regular formula containing intact cow's milk proteins in low-atopy-risk infants has proven to significantly reduce the occurrence of atopic dermatitis compared to infants receiving the control formula [19]. Another promising combination might be a symbiotic mixture, a combination of prebiotics and probiotics. It was shown that infants with atopic dermatitis who received a specific synbiotic mixture containing scGOS/lcFOS combined with Bifidobacterium breve for a period of three months, had a lower prevalence of asthma-like symptoms and asthma medication use at one-year follow-up than the placebo group [20], accompanied with significantly lower levels of cat dander-specific IgE.

The composition of the bifidobacteria and lactobacilli of the children fed the specific scGOS/lcFOS mixture was significantly different, the numbers of both bifidobacteria as well as lactobacilli were increased accompanied by a significant decrease in $\mathrm{pH}$ values of the faeces. The observed manipulation of bifidobacteria and lactobacilli had only slightly influenced the immune response with respect to several biomarkers for the immune system that are well known as predictive biomarkers for atopic dermatitis and/or allergy to food proteins [2124]. In the study described by Moro et al. [4] there was only an increase in the number of bifidobacteria whereas no significant influence on lactobacilli counts was observed.

In allergic syndromes, IgE is thought to have a central role in eliciting immediate hypersensitivity reactions. In this study, $\operatorname{IgE}$ concentrations in the serum samples showed a trend towards a decrease in the prebiotic group. This observation is in line with the data found in infants fed the same prebiotic mixture in an extensively hydrolysed formula. In that study, prebiotic supplementation led to a significant reduction in the plasma level of total IgE [6]. In the current study also a decrease in the percentage of children with elevated $\operatorname{IgE}$ (>15 kU/l) [25] was found, although this difference was not statistically significant. The concentration of $ß$-lactoglobulin-specific IgE was low in both groups and did not show any statistical differences. In order to investigate whether the functionality of the serum IgE was affected, in vitro degranulation assays were performed. However, none of the sera was able to induce degranulation of the RBL cells after stimulation with cow's milk. It might be that for the detection of specific and functional $\operatorname{IgE}$ the infants are simply too young.
Immunoglobulin $\mathrm{G}(\mathrm{IgG})$ antibodies to food allergens are produced in both atopic and non-atopic children. IgG antibody responses to food allergens are observed already at birth and are mainly maternally derived at that age [26]. The children's own IgG antibody production to food allergens peak in early childhood and decline by $8 \mathrm{yr}$ of age. Allergic symptoms and atopic sensitization are associated with high levels of specific IgG subclass antibodies to allergens, particularly IgG4 [27]. Recently it was found that eczematous, food sensitized infants with high levels of IgG4 and high ratios of IgG4/IgE antibodies to food allergens were more likely to consume the allergen-containing food at $4 \frac{1}{2}$ years than infants with low levels and ratios [28]. It was also observed that a combination of high cow's milk-specific IgE and low cow's milk-specific IgG4 levels in infants with cow's milk allergy confirms that the balance between allergen-specific IgE and IgG4 had an impact on whether clinical allergy or tolerance develops [29]. In this study, total and B-lactoglobulin-specific IgG4 was measured and there were no differences in concentration of both total and B-lactoglobulinspecific IgG4 as well as the ratio $\operatorname{IgG} 4 / \operatorname{IgE}$ between the two groups. These observations are in line with the data found in infants fed the same prebiotic mixture in an extensively hydrolysed formula where no effect on total IgG4 was observed [6]

Tetrameric immunoglobulins (Ig) are produced and secreted by plasma cells. However, it is well documented that plasma cells also produce and secrete Ig-free light chain (Ig-fLC) in excess over Igheavy chains [30,31]. Turnover studies in humans demonstrated that only $60 \%$ of synthesized Ig-fLC was incorporated into isotypic whole Ig and that the remaining fraction was released into serum as Ig-fLCs [32]. Ig-fLCs are present in normal serum and can further be detected in urine and cerebrospinal fluid. In several patients suffering from immunological disorders, an increase in Ig-fLC levels can be detected [33] and recently it was demonstrated that Ig-fLCs play a pivotal role in allergic diseases. Ig-fLCs could transfer immediate hypersensitivity-like responses in mice [34] and blocking of Ig-fLC with a specific peptide antagonist prevented the development of contact hypersensitivity in mice [35]. Ig-fLCs were also able to sensitize mast cells [36]. Nasal IgfLC was significantly increased in allergic and nonatopic rhinitis nasal mucosa [37]. Recently, the high relevance of both IgE and Ig-fLC in atopic dermatitis [38] as well as allergy to cow's milk proteins was substantiated [9]. In this current study, no differences were observed in the levels of both kappa Ig-fLC and lambda Ig-fLC between the groups. This in contrast in children receiving scGOS/lcFOS in an extensively hydrolysed formula, where significantly reduced plasma levels of both kappa Ig-fLC and lambda Ig-fLC were observed [39].

A role of gut microbiota in establishing equilibrium between Th1 and Th2 immunological responses has been postulated [40]. It is not yet clear whether gut microbiota and microbial components play a role in the development and function of regulatory $\mathrm{T}$ cells [41]. It is known that a low diversity in the gut microbiota is seen in children with atopic dermatitis [42]. The study of Moro et al. showed that changes in bifidobacteria seem to be linked, at least in part, to changes in atopic dermatitis [19], which is accompanied by a decrease in IgE [6] as well as fLC [43], which are both indications for a reduction of Th2-mediated allergic disorders such as atopic dermatitis. Vos et al. [44] and van't Land et al. [45] showed that scGOS/lcFOS was able to stimulate the Th1 response in an influenza vaccination model in mice. These data indicate that regulatory $\mathrm{T}$ cells might play a role in the modulation of immune responses by modulating the composition of the gut microbiota with prebiotics.

Interestingly, none of the measured biomarkers indicated any specific immunological reaction against cow's milk protein although 
cow's milk proteins were part of the diet. Furthermore, the use of intact protein in the group of infants at risk for allergies with or without prebiotics resulted in a similar cumulative incidence of atopic dermatitis when compared to the incidence in the breast fed group. The supplementation of intact cow's milk proteins to children at risk for allergies does not seem to evoke a strong reaction against cow's milk proteins.

\section{Conclusions}

The data of this exploratory study showed that the scGOS/lcFOS mixture significantly influences the composition of bifidobacteria and lactobacilli. However, the positive immune effects induced by scGOS/ lcFOS as found in infants at risk on extensively hydrolysed formula were only slightly detectable in infants fed intact cow's milk protein containing formula. There were some observations with respect to the immune parameters which need further investigation. We hypothesise that the antigenic protein load is responsible for the less clear effects of scGOS/lcFOS in this study compared to data from infants fed a hydrolysed formula.

\section{Competing Interests}

Knipping, Boehm and Garssen are employees of Danone Research. All other declare that there are no conflicts of interest.

\section{References}

1. Høst A, Koletzko B, Dreborg S, Muraro A, Wahn U, et al. (1999) Dietary products used in infants for treatment and prevention of food allergy. Joint Statement of the European Society for Paediatric Allergology and Clinical Immunology (ESPACI) Committee on Hypoallergenic Formulas and the European Society for Paediatric Gastroenterology, Hepatology and Nutrition (ESPGHAN) Committee on Nutrition. Arch Dis Child 81: 80-84.

2. Osborn DA, Sinn J (2003) Formulas containing hydrolysed protein for prevention of allergy and food intolerance in infants. Cochrane Database Syst Rev: CD003664.

3. Wahn HU (2008) Strategies for atopy prevention. J Nutr 138: 1770S-1772S.

4. Moro G, Arslanoglu S, Stahl B, Jelinek J, Wahn U, et al. (2006) A mixture of prebiotic oligosaccharides reduces the incidence of atopic dermatitis during the first six months of age. Arch Dis Child 91: 814-819.

5. Arslanoglu S, Moro GE, Schmitt J, Tandoi L, Rizzardi S, et al. (2008) Early dietary intervention with a mixture of prebiotic oligosaccharides reduces the incidence of allergic manifestations and infections during the first two years of life. J Nutr 138: 1091-1095.

6. van Hoffen E, Ruiter B, Faber J, M'Rabet L, Knol EF, et al. (2009) A specific mixture of short-chain galacto-oligosaccharides and long-chain fructooligosaccharides induces a beneficial immunoglobulin profile in infants at high risk for allergy. Allergy 64: 484-487.

7. Schouten B, van Esch BC, Hofman GA, van Doorn SA, Knol J, et al. (2009) Cow milk allergy symptoms are reduced in mice fed dietary synbiotics during oral sensitization with whey. J Nutr 139: 1398-1403.

8. Vos AP, M'Rabet L, Stahl B, Boehm G, Garssen J (2007) Immune-modulatory effects and potential working mechanisms of orally applied nondigestible carbohydrates. Crit Rev Immunol 27: 97-140.

9. Schouten B, van Esch BC, van Thuijl AO, Blokhuis BR, Groot Kormelink T, et al. (2010) Contribution of IgE and immunoglobulin free light chain in the allergic reaction to cow's milk proteins. J Allergy Clin Immunol 125: 1308-1314.

10. Harrigan E, Rabinowitz L (1999) Atopic dermatitis. Immunol Allergy Clin North Am 19: 383-396.

11. Muraro A, Dreborg S, Halken S, Host A, Niggemann B, et al. (2004) Dietary prevention of allergic diseases in infants and small children. Part II. Evaluation of methods in allergy prevention studies and sensitization markers. Definitions and diagnostic criteria of allergic diseases. Pediatr Allergy Immunol 15: 196205

12. (1993) Severity scoring of atopic dermatitis: the SCORAD index. Consensus Report of the European Task Force on Atopic Dermatitis. Dermatology 186 23-31.
13. Kunz B, Oranje AP, Labrèze L, Stalder JF, Ring J, et al. (1997) Clinical validation and guidelines for the SCORAD index: consensus report of the European Task Force on Atopic Dermatitis. Dermatology 195: 10-19.

14. Fanaro S, Vigi V, Chierici R, Boehm G (2003) Fecal flora measurements of breastfed infants using an integrated transport and culturing system. Acta Paediatr 92: 634-635.

15. Bousquet J, Chanez P, Chanal I, Michel FB (1990) Comparison between RAST and Pharmacia CAP system: a new automated specific IgE assay. J Allergy Clin Immunol 85: 1039-1043.

16. Takagi K, Nakamura R, Teshima R, Sawada J (2003) Application of human Fc epsilon RI alpha-chain-transfected RBL-2H3 cells for estimation of active serum IgE. Biol Pharm Bull 26: 252-255.

17. Knipping K, van Esch EC, Wijering SC, van der Heide S, Dubois AE, et al (2008) In vitro and in vivo anti-allergic effects of Arctium lappa L. Exp Biol Med (Maywood) 233: 1469-1477.

18. Abe M, Goto T, Kosaka M, Wolfenbarger D, Weiss DT, et al. (1998) Differences in kappa to lambda (kappa:lambda) ratios of serum and urinary free light chains. Clin Exp Immunol 111: 457-462.

19. Grüber C, van Stuijvenberg M, Mosca F, Moro G, Chirico G, et al. (2010) Reduced occurrence of early atopic dermatitis because of immunoactive prebiotics among low-atopy-risk infants. J Allergy Clin Immunol 126: 791-797.

20. van der Aa LB, van Aalderen WM, Heymans HS, Henk Sillevis Smitt J, Nauta AJ, et al. (2011) Synbiotics prevent asthma-like symptoms in infants with atopic dermatitis. Allergy 66: 170-177.

21. García-Ara MC, Boyano-Martínez MT, Díaz-Pena JM, Martín-Muñoz MF Martín-Esteban M (2004) Cow's milk-specific immunoglobulin E levels as predictors of clinical reactivity in the follow-up of the cow's milk allergy infants. Clin Exp Allergy 34: 866-870.

22. Bernardi D, Borghesan F, Faggian D, Bianchi FC, Favero E, et al. (2008) Time to reconsider the clinical value of immunoglobulin G4 to foods? Clin Chem Lab Med 46: 687-690.

23. Ott H, Stanzel S, Ocklenburg C, Merk HF, Baron JM, et al. (2009) Total serum $\lg \mathrm{E}$ as a parameter to differentiate between intrinsic and extrinsic atopic dermatitis in children. Acta Derm Venereol 89: 257-261.

24. Ladics GS, van Bilsen JH, Brouwer HM, Vogel L, Vieths S, et al. (2008) Assessment of three human FcepsilonRl-transfected RBL cell-lines for identifying IgE induced degranulation utilizing peanut-allergic patient sera and peanut protein extract. Regul Toxicol Pharmacol 51: 288-294.

25. Kjellman NM, Johansson SG, Roth A (1976) Serum IgE levels in healthy children quantified by a sandwich technique (PRIST). Clin Allergy 6: 51-59.

26. Jenmalm MC, Björkstén B (2000) Cord blood levels of immunoglobulin G subclass antibodies to food and inhalant allergens in relation to maternal atopy and the development of atopic disease during the first 8 years of life. Clin Exp Allergy 30: $34-40$.

27. Aalberse RC, Schuurman J, van Ree R, Stapel S (1998) IgG4 antibody assays in allergy diagnosis. Res Immunol 149: 263-266.

28. Tomicić S, Norrman G, Fälth-Magnusson K, Jenmalm MC, Devenney I, et al (2009) High levels of IgG4 antibodies to foods during infancy are associated with tolerance to corresponding foods later in life. Pediatr Allergy Immunol 20 : $35-41$

29. Savilahti EM, Saarinen KM, Savilahti E (2010) Specific antibodies to cow's milk proteins in infants: effect of early feeding and diagnosis of cow's milk allergy. Eur J Nutr 49: 501-504

30. Hopper JE, Papagiannes E (1986) Evidence by radioimmunoassay that mitogen-activated human blood mononuclear cells secrete significant amounts of light chain Ig unassociated with heavy chain. Cell Immunol 101: 122-131.

31. Hannam-Harris AC, Smith JL (1981) Free immunoglobulin light chain synthesis by human foetal liver and cord blood lymphocytes. Immunology 43: 417-423.

32. Waldmann TA, Strober W, Mogielnicki RP (1972) The renal handling of low molecular weight proteins. II. Disorders of serum protein catabolism in patients with tubular proteinuria, the nephrotic syndrome, or uremia. J Clin Invest 51 2162-2174.

33. Groot Kormelink T, Thio M, Blokhuis BR, Nijkamp FP, Redegeld FA (2009) Atopic and non-atopic allergic disorders: current insights into the possible involvement of free immunoglobulin light chains. Clin Exp Allergy 39: 33-42. 
Citation: Knipping K, Orsi A, Boehm G, Castoldi F, Garssen J, et al. (2012) Microbial and Immune Biomarkers in Infants at Risk for Allergies Fed an Intact Cow's Milk Formula Containing Specific Non-Digestible Carbohydrates. J Nutr Disorders Ther 2:119. doi:10.4172/2161-0509.1000119

Page 7 of 7

34. Redegeld FA, van der Heijden MW, Kool M, Heijdra BM, Garssen J, et al. (2002) Immunoglobulin-free light chains elicit immediate hypersensitivity-like responses. Nat Med 8: 694-701.

35. van Houwelingen AH, Kaczynska K, Kraneveld AD, Kool M, Nijkamp FP, et al. (2007) Topical application of F991, an immunoglobulin free light chain antagonist, prevents development of contact sensitivity in mice. Clin Exp Allergy 37: 270-275.

36. Redegeld FA, Wortel $\mathrm{CH}$ (2008) IgE and immunoglobulin free light chains in allergic disease: new therapeutic opportunities. Curr Opin Investig Drugs 9 : $1185-1191$.

37. Powe DG, Groot Kormelink T, Sisson M, Blokhuis BJ, Kramer MF, et al. (2010) Evidence for the involvement of free light chain immunoglobulins in allergic and nonallergic rhinitis. J Allergy Clin Immunol 125: 139-145.

38. Kayserova J, Capkova S, Skalicka A, Vernerova E, Polouckova A, et al. (2010) Serum immunoglobulin free light chains in severe forms of atopic dermatitis. Scand J Immunol 71: 312-316.

39. Schouten B, Van Esch BC, Kormelink TG, Moro GE, Arslanoglu S, et al. (2011) Non-digestible oligosaccharides reduce immunoglobulin free light-chain concentrations in infants at risk for allergy. Pediatr Allergy Immunol 22: 537 542 .
40. Neurath MF, Finotto S, Glimcher LH (2002) The role of Th1/Th2 polarization in mucosal immunity. Nat Med 8: 567-573.

41. Hrncir T, Stepankova R, Kozakova H, Hudcovic T, Tlaskalova-Hogenova $H$ (2008) Gut microbiota and lipopolysaccharide content of the diet influence development of regulatory T cells: studies in germ-free mice. BMC Immunol 9: 65.

42. Abrahamsson TR, Jakobsson HE, Andersson AF, Björkstén B, Engstrand L, et al. (2012) Low diversity of the gut microbiota in infants with atopic eczema. $J$ Allergy Clin Immunol 129: 434-440

43. Schouten B, Van Esch BC, Groot Kormelink TG, Moro GE, Arslanoglu S, et al. (2011) Non-digestible oligosaccharides reduce immunoglobulin free lightchain concentrations in infants at risk for allergy. Pediatr Allergy Immunol 22: 537-542.

44. Vos AP, Haarman M, Buco A, Govers M, Knol J, et al (2006) A specific prebiotic oligosaccharide mixture stimulates delayed-type hypersensitivity in a murine influenza vaccination model. Int Immunopharmacol 6: 1277-1286.

45. van't Land B, Schijf M, van Esch BC, van Bergenhenegouwen J, Bastiaans J et al. (2010) Regulatory T-cells have a prominent role in the immune modulated vaccine response by specific oligosaccharides. Vaccine 28: 5711-5717. 